\title{
Nosocomial infections: knowledge and source of information among clinical health care students in Ghana
}

This article was published in the following Dove Press journal:

International Journal of General Medicine

10 August 2011

Number of times this article has been viewed

\author{
Ajediran I Bello' \\ Eunice $\mathrm{N}$ Asiedu' \\ Babatunde OA Adegoke ${ }^{2}$ \\ Jonathan NA Quartey' \\ Kwadwo O Appiah-Kubi' \\ Bertha Owusu-Ansah' \\ 'Department of Physiotherapy, School \\ of Allied Health Sciences, College \\ of Health Sciences, University of \\ Ghana, Accra, Ghana; ${ }^{2}$ Department of \\ Physiotherapy, College of Medicine, \\ University of Ibadan, Ibadan, Nigeria
}

Background: This study determined and compared the knowledge of nosocomial infections among clinical health care students at the College of Health Sciences, University of Ghana.

Methods: Two hundred undergraduate health care students from four academic programs participated in the study. The study sample was drawn from each academic program by a simple random sampling technique using the class directory from each course. The Infection Control Standardized Questionnaire (ICSQ) was used to assess the knowledge of students about three main domains, ie, hand hygiene, nosocomial infections, and standard precautions. A maximum score of 50 was obtainable, and respondents with scores $\geq 70 \%$ were classified as having a satisfactory knowledge. The response on each item was coded numerically to generate data for statistical analysis. Comparison of knowledge on the domains among categories of students was assessed using the Kruskal-Wallis test, while associations between courses of study and knowledge about nosocomial infections were determined using the Chi-square test. All statistical tests had a significant level of 5\% $(P<0.05)$

Results: Overall mean percentage score of the participants on ICSQ was $65.4 \pm 2.58$, with medical, physiotherapy, radiography, and nursing students recording mean percentage scores of $70.58 \pm 0.62,65.02 \pm 2.00,64.74 \pm 1.19$, and $61.31 \pm 2.35$, respectively. The main source of information about the prevention of nosocomial infections as cited by participants was their routine formal training in class. There was no significant association $(P>0.05)$ between course of study and knowledge of students about preventive measures for nosocomial infections.

Conclusion: The students sampled demonstrated moderate knowledge of nosocomial infections and this was acquired largely through formal classroom training. These findings underscore the need for more emphasis on education about this important source of infection in the clinical training curriculum.

Keywords: knowledge, prevention, nosocomial infections

\section{Introduction}

Health care-associated infections have long been recognized as crucial factors bedeviling the quality and outcomes of health care delivery. ${ }^{1}$ An infection is considered nosocomial if it becomes evident 48 hours or more after hospital admission or within 30 days of discharge following inpatient care. ${ }^{2}$ Reported cases of nosocomial infection assumed such terrifying proportions in 2002 that World Health Organization member states approved a World Health Assembly resolution on patient safety. ${ }^{3}$ Developing countries were reported to have up to 20 times the risk of contracting a nosocomial infection compared with developed countries. ${ }^{3}$ Thus, spread of infection serves as a major source of worry for managers in health care practice, particularly in developing countries where the health care system is already overstretched.
Correspondence: Ajediran I Bello Department of Physiotherapy, College of Health Sciences, University of Ghana, Accra, Ghana

Tel +233249294930

Fax +2332 |68 829।

Email badmus@chs.edu.gh 
Although infection is most prevalent in patients upon admission, health care workers also act as potential vectors for pathogenic agents. Hospitals provide a favorable transmission pathway for the spread of nosocomial infections, owing partly to poor infection control practices among health workers on one hand and overcrowding of patients in most clinical settings on the other. ${ }^{2}$ The importance of hospital-acquired infections goes beyond its impact on morbidity and mortality figures in any country, and has profound economic implications. According to Robert, acquisition of a nosocomial infection can prolong duration of hospitalization, increase the costs of health care, and place a serious economic burden on patients and their families. ${ }^{4}$

This scenario should alert clinical instructors and supervisors to the need to pay adequate attention to imparting knowledge to students throughout their training period about measures to prevent nosocomial infections. ${ }^{5}$ In one survey, $27 \%$ of participating health care students reported insufficient emphasis on teaching about infection control in their training program, whilst 50\% expressed a desire for more emphasis on isolation procedures during their training. ${ }^{6}$

Renewed effort geared towards education in terms of training and retraining about standard infection control, as well as strict adherence by health care staff and students to aseptic practice, can reduce the extent of these risks. ${ }^{7}$ Considering the lack of information describing students' knowledge of the preventive measures necessary to limit the spread of nosocomial infections, this study provides insight into knowledge about nosocomial infection among health care students undertaking different training programs in Ghana.

\section{Methodology}

\section{Participants}

The study was carried out at the College of Health Sciences, University of Ghana, located at the Korle-Bu Teaching Hospital in Accra. This health care training institution is made up of seven schools which train various medical and allied health care professionals. The research was a cross-sectional survey. Students were selected from each academic program using a simple random sampling technique by ballot. This was done by requesting the directory for each class. Using this process, 200 students from physiotherapy, radiography, medicine, and nursing who were in their first- and secondyear clinical phase were selected for study.

\section{Survey instruments}

The Infection Control Standardized Questionnaire (ICSQ) ${ }^{8}$ prepared by infection control experts according to international guidelines on standard isolation precautions and hand hygiene was used in this study. The questionnaire consisted of three main domains, with 25 close-ended items, ie, students' knowledge about nosocomial infection (five items), standard precautions (12 items), and hand hygiene (eight items). Response to each item was coded and scored as a correct answer (2), don't know (1), or incorrect answer (0). The instrument was found to be internally consistent, with a Cronbach test score of 0.61 . We used a data capturing form to collect data on the subjects' sociodemographic characteristics, their definition of nosocomial infection, and their main source of information.

\section{Procedure}

Prior to the study, participants were given a brief introduction to the purpose of the study, after which their consent was sought and obtained. Ethical approval for this research was obtained from the ethical review committee of the School of Allied Health Sciences, College of Health Sciences, University of Ghana. All the participants completed and returned the questionnaire, giving a response rate of $100 \%$.

\section{Statistical analysis}

All returned copies of the questionnaire were analyzed using the Statistical Package for Social Sciences (SPSS Inc, Chicago, IL) version 16. The maximum possible score obtainable was 50 in all three domains. Respondents who scored $35(70 \%)$ or more were rated as having satisfactory knowledge on the subject. Descriptive statistics were used to summarize the data obtained. Association between courses of study and knowledge about nosocomial infections was tested using the Chi-square test, and the Kruskal-Wallis test to compare differences in the knowledge between the different student groups. All statistical tests had a significant level of $5 \%(P<0.05)$.

\section{Results Profiles of participants}

Two hundred clinical students were randomly sampled to participate in the study. They comprised 102 males $(51 \%)$ and 98 females (49\%). Their mean age was $23.5 \pm 1.20$ years. Seventy participants each were sampled from nursing students and medical students, whilst 30 each were sampled from physiotherapy and radiography students. A response rate of $100 \%$ was achieved through close follow-up and the personal interest in nosocomial infection among the participants. 


\section{Knowledge distribution}

The overall mean percentage score for the standardized infection control question was $65.4 \pm 2.1$. Medical students recorded a mean score of $70.58 \pm 0.62$, physiotherapy students scored $65.02 \pm 2.07$, radiography students scored $64.74 \pm 1.19$, and nursing students scored $61.31 \pm 2.35$ (Table 1). Comparison of mean percentage scores among the four programs using the Kruskal-Wallis test did not show any statistically significant difference $(P>0.05)$ in knowledge about hand hygiene domains. However, there was a statistically significant difference in mean percentage scores $(P=0.024)$ in the standard precautions domain of the ICSQ between the different student categories. The main source of information for most students was formal training in class, cited by 76 students, representing $38 \%$ of the sampled population.

\section{Course of study and knowledge about prevention}

The students were assessed on the basis of their knowledge in relation to their courses of study. No statistical significant association $(P>0.05)$ was found between their courses of study and knowledge on the prevention of nosocomial infection on the three domains.

\section{Discussion}

The study identified and compared knowledge about nosocomial infection and sources of information among 200 health care students. There was no significant difference on the knowledge and hand hygiene domain scores between the student categories, whereas a significant difference was found in the standard precautions domain, where medical students scored highest. Formal training in class was also identified as the main source of information.

Awareness and background knowledge about nosocomial infections among the selected students were moderate and was most evident among medical students. Formal training (36\%) in class was shown to be the main source of information influencing students' knowledge about preventive measures for nosocomial infections. This proportion was relatively low compared with that obtained in a study by Tavolacci et al, in which $92 \%$ of sampled students citing formal training in class as their main source of information. ${ }^{8}$ This disparity in outcome may be due to methodological and environmental factors. Whilst a total of 200 hundred students participated in the present study, the previous study involved 350 students. Also, the study location might have influenced the differences in outcome of the two studies. Nevertheless, the highest reference made to formal training in both studies was suggestive of a more theoretical approach to campaign about nosocomial infection control.

Although there were differences in the standard precautions knowledge domain between the four academic programs, there was no statistically significant association between the course of study and knowledge amongst the students sampled. This implies that, although the students recorded different overall percentage mean scores in the domains of the questionnaire, this was not statistically different enough to account for knowledge acquisition on the basis of course of study. The performance of each student category also varied with the domains. For instance, while the highest mean scores were recorded by medical students for the infections and standard precautions domain, physiotherapy students had the highest score on the hand hygiene domain but recorded the lowest score for the nosocomial infection domain. This outcome followed the same trend as that reported by Suchitra and Lakshimi, although the difference in the present study was not substantial enough to be of statistical significance. ${ }^{6}$

Further, the knowledge of the students about hand hygiene in the prevention of nosocomial infection did not show any statistically significant difference between the training courses. This outcome seems to be at variance with findings by Tavolacci et al, who reported that nursing students expectedly had the highest score on all three domains of the questionnaire. ${ }^{8}$ The recruitment of degree nursing students who are more theoretically inclined rather than regular

Table I Kruskal-Wallis test comparison of knowledge of different student groups on nosocomial infection, standard precautions, and hand hygiene

\begin{tabular}{|c|c|c|c|c|c|c|}
\hline Variable & $\begin{array}{l}\text { Physiotherapy } \\
\text { mean \% }\end{array}$ & $\begin{array}{l}\text { Radiotherapy } \\
\text { mean \% }\end{array}$ & $\begin{array}{l}\text { Nursing } \\
\text { mean \% }\end{array}$ & $\begin{array}{l}\text { Medicine } \\
\text { mean \% }\end{array}$ & $\mathbf{H}$ & $P$ value \\
\hline Nosocomial & $45.5 \pm 2.7$ & $49.4 \pm 1.4$ & $47.6 \pm 2.0$ & $64.8 \pm 1.9$ & I.0I I & 0.798 \\
\hline Standard precaution & $87.3 \pm 1.9$ & $83.2 \pm 2.6$ & $79.9 \pm 1.9$ & $90.3 \pm 1.1$ & 14.410 & $0.024^{\mathrm{a}}$ \\
\hline Hand hygiene & $62.0 \pm 1.1$ & $61.6 \pm 2.0$ & $56.6 \pm 2.5$ & $56.6 \pm 1.1$ & 0.713 & 0.870 \\
\hline Total & $65.0 \pm 2.1$ & $61.7 \pm 2.0$ & $61.3 \pm 2.3$ & $70.6 \pm 0.6$ & & \\
\hline
\end{tabular}

Note: $\mathrm{a} P<0.05$ 
nursing students might have accounted for the difference seen in the previous study. This implies a need to strengthen the nursing curriculum on infection control, given that nurses are likely to have more contact with patients than any other health care professionals. It is worth noting that many factors potentially influenced the response of participants in this study, ranging from different levels of entry into the clinical phase of each program, extent of physical contact with patients, training in hands-on techniques, and general patient handling practices.

In conclusion, the results of this study demonstrate that knowledge of nosocomial infection isolation precautions was moderate among most of the health care students sampled. Nonetheless, this calls for a review of health care curricula to pave the way for more pragmatic infection control teaching in all our health care programs.

\section{Disclosure}

The authors report no conflicts of interest in this work.

\section{References}

1. Horan TC, Gaynes RP. Surveillance of nosocomial infections. In: Hospital Epidemiology and Infection. 3rd ed. Mayall CG, editor. Philadelphia, PA: Lippincott, Williams \& Wilkins; 2004.

2. Samuel SO, Kayode OO, Musa OI, et al. Nosocomial infections and the challenges of control in developing countries. Afr J Clin Exp Microbiol. 2009;11:102-110.

3. World Health Organization. 10 facts on patient safety. Available from: http://www.who.int/feature/factsfile/patientsafety/en/index.html. Accessed October 10, 2008.

4. Robert AW. Controlling anti-microbial resistance in hospitals infection control and use of antibiotics. Emerg Infect Dis. 2001;7:188-192.

5. Bryce EA, Scharf S, Walker M, Walsh A. The infection control audit: the standardised audit as a tool for change. Am J Infect Control. 2007; $35: 271-283$.

6. Suchitra JB, Lakshmi Devi N. Impact of education on knowledge, attitudes and practices among various categories of health care workers on nosocomial infections. Indian J Med Microbiol. 2007;25: $181-187$.

7. Philips G, Ker J. Champion students! experience with a standardised infection control training package in medical students. J Hosp Infect. 2006;62:518-519.

8. Tavolacci MP, Ladner J, Bailly L, et al. Prevention of nosocomial infection and standard precautions: knowledge and source of information among healthcare students. Infect Control Hosp Epidemiol. 2008;29: 642-647.

\section{Publish your work in this journal}

The International Journal of General Medicine is an international, peer-reviewed open-access journal that focuses on general and internal medicine, pathogenesis, epidemiology, diagnosis, monitoring and treatment protocols. The journal is characterized by the rapid reporting of reviews, original research and clinical studies across all disease areas.
A key focus is the elucidation of disease processes and management protocols resulting in improved outcomes for the patient. The manuscript management system is completely online and includes a very quick and fair peer-review system. Visit http://www.dovepress.com/ testimonials.php to read real quotes from published authors. 